\title{
Preliminary Exploration Of Theory And Practice Training Of 5G Ultrasonic Remote Consultation In The Grassroot Hospitals
}

\section{Ceng Wang}

Zhejiang Provincial People's Hospital

\section{YI Zheng}

Zhejiang Provincial People's Hospital

Cui Xiong

Zhejiang Provincial People's Hospital

Jing Wang ( $\nabla$ wangjing@hmc.edu.cn )

Zhejiang Provincial People's Hospital

\section{Research Article}

Keywords: The grassroot hospitals, Ultrasonic remote consultation, Visual apparatus

Posted Date: February 14th, 2022

DOI: https://doi.org/10.21203/rs.3.rs-1295987/v1

License: (a) (i) This work is licensed under a Creative Commons Attribution 4.0 International License. Read Full License 


\section{Abstract}

Background: Many people in rural areas have not had an ultrasound examination for a long time due to lack of medical resources. So we go through training doctors in grassroot hospitals to explore the clinical and practical value of remote ultrasound.

Methods: The Superior hospital will conduct ultrasonic theory and practice training for the grassroot hospitals. After passing the training, remote ultrasonic diagnosis will be carried out at the grassroot level, then feedback and case data will be collected.

Results: Through training, the ultrasonic diagnosis level of the grassroot doctors has been significantly improved. At present, a total of 148 cases of consultation have been completed through remote ultrasound "visual apparatus", mainly abdominal ultrasound, and the common cases are fatty liver, hepatic cyst, gallbladder stone, kidney stone and so on .

Conclusion: As a "visual apparatus", remote ultrasound can be routinely carried out in the grassroot hospitals, which can promote mutual benefit between doctors and patients and comprehensively promote the construction of healthy villages.

\section{Background}

With the rapid development of science and technology, remote diagnosis and treatment system has gradually attracted more and more attention and applications.As an important branch of remote diagnosis and treatment, $5 \mathrm{G}$ ultrasound can greatly improve the level of ultrasonic diagnosis in mountainous areas ${ }^{[1]}$, islands and other areas where medical resources are relatively scarce. Following with the comprehensive application of it, the villagers can directly realize face-to-face remote consultation with expert doctors in community hospitals, Instead of rushing to provincial hospitals and waiting in line for registration. $5 \mathrm{G}$ ultrasound is convenient to get the diagnosis and advice from the provincial ultrasound experts, saving time and economic cost, as well as enjoying the high-quality ultrasound medical resources. As early as 2017,starting from Pengbu Health Center,Jianggan District, Hangzhou, our hospital has officially carried out ultrasonic remote consultation project with many grassroots hospitals, such as Haining, Chun 'an and Taishun. This paper discusses the effect of theory and practice remote ultrasound training in the basic hospital.

\section{Methods}

The relevant materials of theoretical and practical training conducted in the medical community of Taishun County People's Hospital from October 2020 to now were selected. The theoretical training content is shown in Table 1.

Table $1 \otimes T h e$ theoretical training 


\begin{tabular}{|cll|}
\hline 1 & Content & Knowledge points to master \\
\hline 2 & $\begin{array}{l}\text { Principle and application of ultrasound } \\
\text { medicine }\end{array}$ & The application range of ultrasound \\
\hline 3 & $\begin{array}{l}\text { Standard image for conventional ultrasound } \\
\text { applications }\end{array}$ & $\begin{array}{l}\text { Remember the anatomy of the standard } \\
\text { section }\end{array}$ \\
\hline 4 & $\begin{array}{l}\text { Ultrasonographic assessment of trauma } \\
\text { abdomen }\end{array}$ & $\begin{array}{l}\text { Key points of ultrasound assessment in } \\
\text { trauma }\end{array}$ \\
\hline 5 & $\begin{array}{l}\text { Ultrasonographic assessment of } \\
\text { shock/hypoxemia }\end{array}$ & $\begin{array}{l}\text { Key points of ultrasound assessment in acute } \\
\text { abdomen } \\
\text { Key points of ultrasound assessment in } \\
\text { shock/hypoxemia }\end{array}$ \\
\hline
\end{tabular}

At the beginning of the practice training, the handheld ultrasound was carried out under the guidance of the provincial hospital. The practice training content is shown in Table 2.

Table 2:The practice training

\begin{tabular}{|c|c|c|}
\hline & Content & Knowledge points to master \\
\hline \multirow[t]{3}{*}{1} & The routine section of liver & $\begin{array}{l}\text { a.The sagittal section of the left lobe of liver through abdominal } \\
\text { aorta, }\end{array}$ \\
\hline & & $\begin{array}{l}\text { b.The oblique section of right subcostal side through the first } \\
\text { porta of liver, }\end{array}$ \\
\hline & & $\begin{array}{l}\text { c. The oblique section of the costal margin through the second } \\
\text { porta of liver, etc. }\end{array}$ \\
\hline 2 & $\begin{array}{l}\text { The longitudinal section of } \\
\text { gallbladder }\end{array}$ & Measure the maximum diameter of the gallbladder \\
\hline 3 & The section of spleen & Measuring the thickness of spleen $\rrbracket$ through the hilum of spleen $\nabla$ \\
\hline 4 & The section of both kidneys & 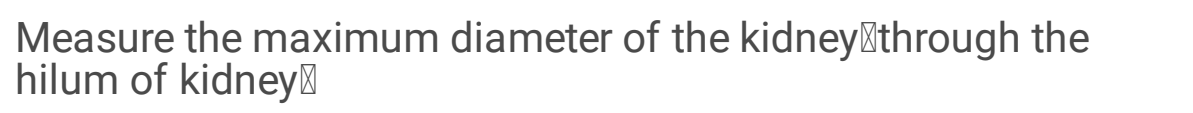 \\
\hline \multirow[t]{5}{*}{5} & The FAST process & $\begin{array}{l}\text { a.The four-chamber heart below the xiphoid process shows the } \\
\text { pericardial cavity, }\end{array}$ \\
\hline & & b.The section of the crypt of the liver and kidney, \\
\hline & & c.The section of the crypt of the spleen and kidney, \\
\hline & & d.The section of the pelvic cavity of the lower abdomen, \\
\hline & & e.The section of the bilateral chest cavity, etc. \\
\hline
\end{tabular}

The content of the theory test is the knowledge in class. After each skill training, 5 specific sections are required to be completed within 5 minutes, and the practice results are counted from the aspects of 
image quality, technique, special parts annotation and proficiency ${ }^{[2]}$, The two teachers graded and averaged the scores. The doctors who have passed theoretical and practical training with the training certificate are required to provide 5 cases per month. After the completion of the training, the doctors was required to fill in the questionnaire to investigate the corresponding problems in the "Feedback Evaluation Form of Taishun Ultrasound Ability Improvement Training Course". The training satisfaction level ranged from 0 to 10 points.

Using Stroke Remote ultrasonic and remote platform, it has the function of real-time remote transmission of ultrasonic images and two-way voice call. The image can be stored on a remote platform for consultation experts to comment and analyze.

Taishun County People's Hospital and 12 affiliated health hospitals were selected as the training objects of this remote consultation.All materials uploaded to the database to provide a platform for learning and exchange. The flow chart of remote consultation was shown in the figure1 below.

\section{Statistics}

SPSS 19.0 statistical software was used, and the enumeration data were expressed by quantity and percentage. Measurement data were described with means \pm standard $(M \pm S D)$, and $t$ test was used for comparison between two independent samples. $P<0.05$ was considered statistically significant.

\section{Results}

\section{Record of practical assessment and theoretical training results}

The training class has 2 practical assessment and 1 theoretical assessment, with a total score of 50 points for each assessment. The difference in scores between the two practical training was statistically significant. See table 3 for relevant results.

Table 3:

\begin{tabular}{lll} 
Practical Training 1 & Practical Training 2 & Theoretical results \\
\hline $25.44 \pm 6.81$ & $40.81 \pm 8.15^{\star}$ & $34.59 \pm 10.97$
\end{tabular}

*shows $P<0.05$

\section{Feedback of trainees after the training}

43 questionnaires were sent out and all questionnaires were collected after the training. 0-5 dissatisfied, 6-7 basically satisfied, 8-10 very satisfied. Satisfaction scores are shown in the table 4.

Table 4: 


\begin{tabular}{ll} 
Satisfaction score (points) & Number of graders (persons) \\
\hline 10 & 35 \\
\hline 9 & 5 \\
\hline 8 & 2 \\
\hline 7 & 1 \\
\hline & 0 \\
1. and the following &
\end{tabular}

\section{Remote consultation cases}

At present, there are 148 cases of consultation cases, including 67 males and 81 females, aged from 21 to 101 years old $(62.40 \pm 15.73)$. There were 47 cases of liver diseases (fatty liver, hepatic cyst, hepatic hemangioma, etc.), 16 cases of gallbladder diseases (cholecystitis, gallstone, polyp, etc.), and 29 cases of urinary diseases (urinary calculi, hydronephrosis, prostatic hyperplasia, bladder wall coarser, etc.). Other relevant information is shown in the following figures, including figure 2:The proportion of remote consultation site; figure 3: The proportion of the most recent ultrasound review at different time periods; figure 4: The proportions of different treatment measures.

\section{Discussion}

China has increased the development of primary medical and health undertakings, and the supporting hardware equipment has also kept pace with the demand for diagnosis and treatment. However, there is still a shortage of primary medical personnel, especially experienced sonographers. Some clinicians are part-time sonographers. Due to lack of systematic theoretical knowledge training and clinical practice operation ${ }^{[3]}$, it is easy to miss diagnosis and misdiagnosis, and the quality of ultrasound examination is greatly reduced, which has a negative impact on grassroots hospitals. A lot of patients, even after seeing common diseases in a grassroots hospital, then still question the diagnosis result and then go to a higher hospital for repeated examination, which consumes precious time, increases the economic burden, and causes a waste of medical resources.

In the past, the methods to improve the level of ultrasonic diagnosis and treatment at the grassroots level mainly include: grassroots doctors go to higher hospitals for further study; The superior hospital visits the basic level and lectures; Formulate the ultrasonic inspection specification, and the quality control center conducts regular inspection. However, due to lack of human resources in primary hospitals, the study time is short and progress is slow. Superior doctors teach and give lectures in basic hospitals, but they only stay at the theoretical level and the scope is limited. The formulation of ultrasonic diagnosis and treatment standards can improve the level of ultrasonic diagnosis and treatment at the grassroots level to a certain extent, but it has little effect on the development of grassroots ultrasonic medicine at the early stage. 
The training is initiated by the Department of Ultrasound of Zhejiang Provincial People's Hospital and the Vocational Training Center for Ultrasound Medicine of Zhejiang Province, based on the 12 grassroots health centers of the Taishun County People's Hospital. The establishment of $5 \mathrm{G}$ ultrasound remote consultation mode between provincial-level Grade A hospitals and grassroots hospitals -- relying on the information system of county, town, village and first aid linkage ultrasonic comprehensive service station - can better serve grass-roots patients ${ }^{[4-5]}$. Since planning training, the instructor has understood the characteristics and requirements of local medicine in advance, and has taken "how to use handheld ultrasound quickly and accurately" as the teaching goal. The theoretical training focuses on the basic principle and usage method of the handheld ultrasound, and the most practical and most urgently needed of ultrasonic knowledge for the grassroots doctors. Practice training students are divided into small groups "hand in hand teaching". After passing the theoretical and practical assessment, the students' practical scores were significantly improved. After obtaining the training certificates, they carried out the diagnosis and treatment work in their respective areas. Through the feedback of students and their suggestions on our training course, we found that: (1) Students believe that this type of training is very practical, but the class are compact and the system of knowledge points is huge. They hope that the class can be extended to give enough time for digestion. (2)The theoretical courses is difficult, so the principles of ultrasound physics can be appropriately deleted. For general practitioners who have no ultrasound foundation, they prefer to have quick and simple introductory courses. (3) Instructor responsibility system is implemented in training, with one tutor leading 2-3 students. Small class teaching are optimized, and there is enough training time to refine to each aspect, and pass the test one by one.

At present, we have completed a total of 148 cases of consultation through the remote ultrasound "Visualization apparatus". Abdominal ultrasound was the main method囚mainly fatty liver, liver cyst, gallstone, kidney stone. Due to the weak health awareness and lack of medical resources at the grassroots level, some patients have not ultrasound examination for more than 5 years. "Visualization apparatus" as a newly developed thing, different from static images such as radiation and electrocardiogram, real-time dynamic scanning is very important. The quality of the images is largely determined by the manipulation, and clearer and more comprehensive images will lead to more accurate diagnosis. Provincial experts can observe the size, shape, peripheral tissue relationship and blood flow signal of lesions in real time through the remote consultation system, and guide the operation techniques of grassroots doctors ${ }^{[6-7]}$. On the basis of voice and video, experts can not only communicate with patients to ask for a brief history, but also make simple visual examination, such as body mass, location of pain and other relevant auxiliary information. In addition, through real-time explanation and guidance from experts, grassroots doctors can greatly improve the diagnostic coincidence rate. This process is also conducive to the expansion of their own diagnostic ideas and the improvement of the accuracy of operation techniques ${ }^{[8]}$, so as to attract patients to seek medical treatment. Common cases can be solved in grassroots hospitals, which is conducive to the development of hierarchical diagnosis and treatment in the grassroot hospitals. There are also relevant literature reports that $5 \mathrm{G}$ remote ultrasound can be used to examine COVID-19 patients, transmit images to experts outside the isolation area, which can not only 
reduce the contact of medical staff, but also obtain better diagnosis and treatment resources, with good clinical and scientific research value.

\section{Conclusion}

To sum up, ultrasonic remote consultation can not only facilitate grassroots patients and realize hierarchical diagnosis and treatment, but also promote the improvement of the clinical level and ultrasonic skills of grassroots doctors ${ }^{[9-10]}$. With the development of $5 \mathrm{G}$ network and telemedicine on the right track, remote ultrasound, as a "Visual apparatus", has been routinely carried out in grassroots hospitals. The effect will be in all aspects: mutual benefit between doctors and patients, improvement of grassroots infrastructure conditions, improvement of disease prevention, treatment and health management capabilities. And all of them form a grassroots medical community system, and promote overall healthy village construction. At the same time, in the current epidemic of COVID-19, we can use remote ultrasound and robots to examine infected people, reduce transmission routes and avoid infection of health care workers, which can also be used as a research.

\section{Declarations}

Ethics approval and consent to participate $\triangle A l l$ experiments were performed in accordance with relevant guidelines and regulations (Declaration of Helsinki). All experimental protocols were approved by the Ethics Committee of Zhejiang Provincial People's Hospital. The informed consent was obtained from all subjects.

Consent for publication $₫$ Consent for publication was obtained for every individual person's data included in the study.

Availability of data and materials: All data generated or analysed during this study are included in this published article,The datasets generated and analysed during the current study are not publicly available due to The data may be applied to other relevant studies but are available from the corresponding author on reasonable request.

Competing interests $\otimes T$ The authors declare that they have no conflict of interest.

Funding: Zhejiang Provincial Health Science and Technology Project Plan(2021KY062) and (2022KY565)

Authors' contributions $\llbracket$ Ceng Wang and Jing Wang conceived and designed the study. Yi Zheng and Cui Xiong looked up relevant information. Ceng Wang wrote the paper. Jing Wang, Yi Zheng and Cui Xiong reviewed and edited the manuscript. All authors read and approved the manuscript.

Acknowledgements $\otimes$ First of all, I would like to extend my sincere gratitude to my partener, Jing Wang, for her instructive advice and useful suggestions on my thesis. I am deeply grateful of her help in the completion of this thesis. Then I am also deeply indebted to Yi Zheng and Cui Xiong in Translation 
Studies for their direct and indirect help to me.Special thanks should go to these partners who have put considerable time and effort into their comments on the draft. Finally, I am indebted to my parents for their continuous support and encouragement.

\section{Reference}

1. Karako K, Song P, Chen Y, Tang W. Realizing 5G- and Al-based doctor-to-doctor remote diagnosis: opportunities, challenges, and prospects. Biosci Trends. 2020 Nov 4;14(5):314-317.

2. Shaffer M, Brown HA, McCoy C, Bashaka P. Evaluation of a Short-term Training Program in Bedside Emergency Ultrasound in Southwestern Tanzania. J Ultrasound Med. 2017 Mar;36(3):515-521.

3. Denny SP, Minteer WB, Fenning RTH, et al. Ultrasound curriculum taught by first-year medical students: A four-year experience in Tanzania. World J Emerg Med. 2018;9(1):33-40.

4. Ye R, Zhou X, Shao F, et al. Feasibility of a 5G-Based Robot-Assisted Remote Ultrasound System for Cardiopulmonary Assessment of Patients With Coronavirus Disease 2019. Chest. 2021 Jan;159(1):270-281.

5. Wang J, Peng C, Zhao Y, et al.Application of a Robotic Tele-Echography System for COVID-19 Pneumonia. J Ultrasound Med. 2021 Feb;40(2):385-390.

6. Salerno A, Kuhn D, El Sibai R, et al. Real-Time Remote Tele-Mentored Echocardiography: A Systematic Review. Medicina (Kaunas). 2020 Dec 2;56(12):668.

7. Adams SJ, Burbridge BE, Badea A, et al. A crossover comparison of standard and telerobotic approaches to prenatal sonography. J Ultrasound Med 2018; 37:2603-2612.

8. Prosch H, Radzina M, Dietrich CF, et al.Ultrasound Curricula of Student Education in Europe: Summary of the Experience. Ultrasound Int Open. 2020 Jun;6(1):E25-E33.

9. Shah S, Tohmasi S, Frisch E, et al. A comparison of simulation versus didactics for teaching ultrasound to Swiss medical students. World J Emerg Med. 2019;10(3):169-176.

10. Hempel D, Schröper T, Pfister R, Michels G. Sonographieausbildung in der Notfall- und Intensivmedizin : Integration schon im Medizinstudium? [Ultrasound training in emergency and intensive care medicine : Integration already in medical school?]. Med Klin Intensivmed Notfmed. 2019 Sep;114(6):519-524.

\section{Figures}




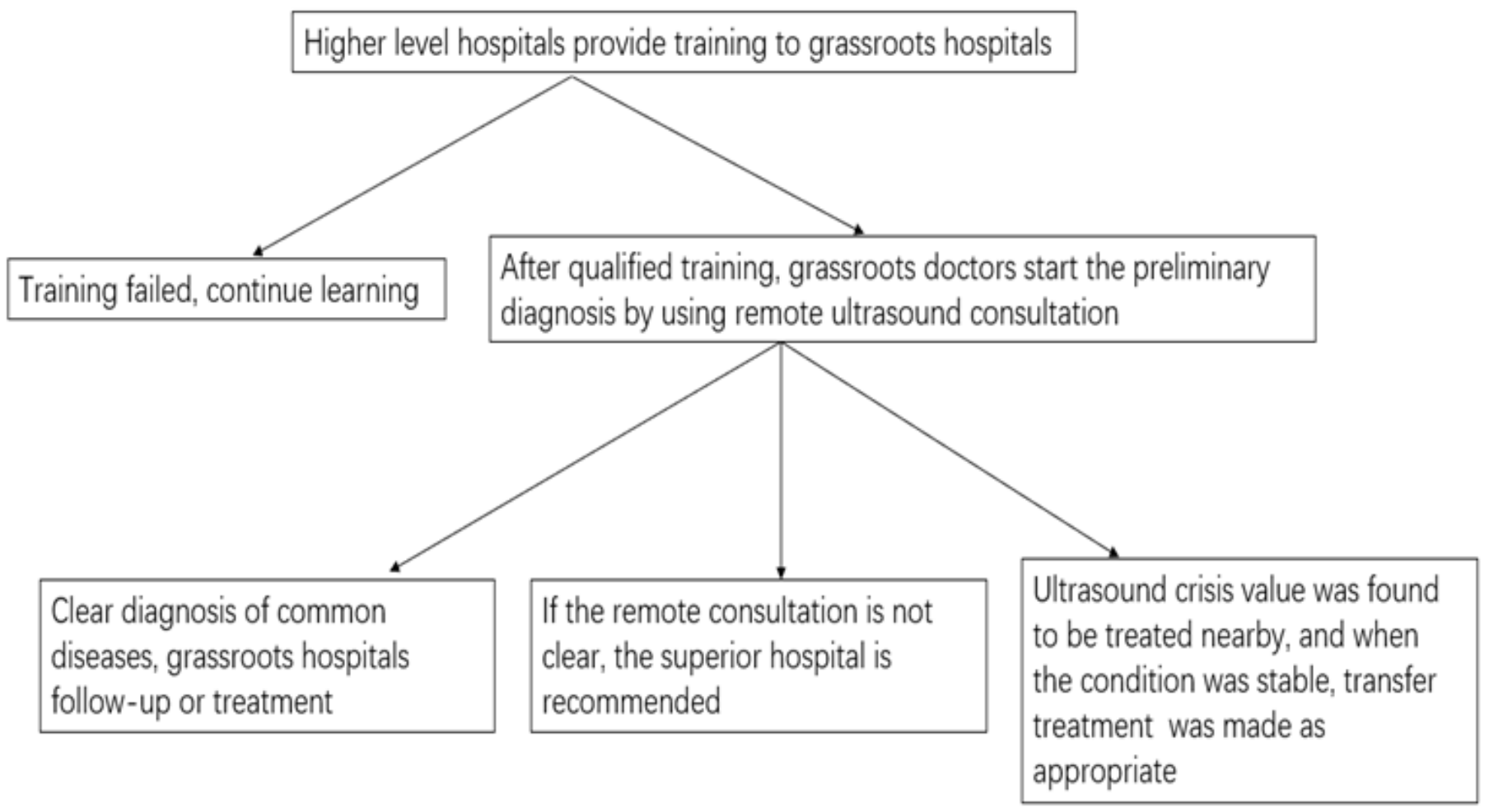

\section{Figure 1}

The flow chart of remote consultation

\section{Remote Consultation Site}

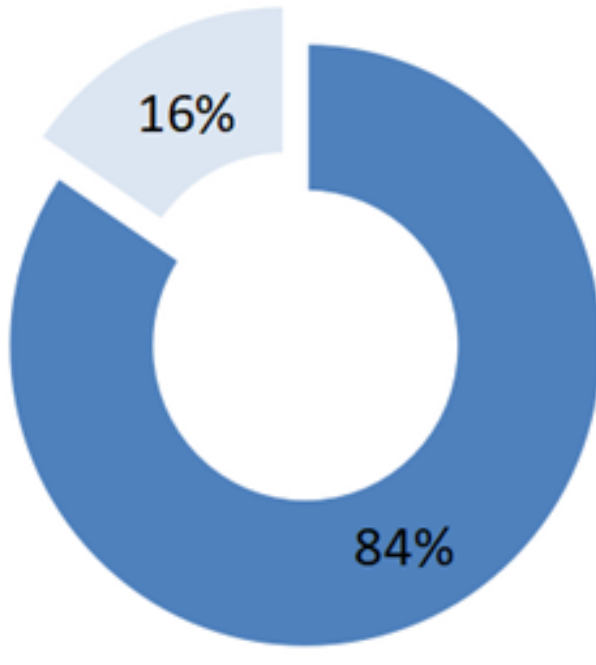

- outpatient $=$ Non-outpatient

\section{Figure 2}


The proportion of remote consultation site.

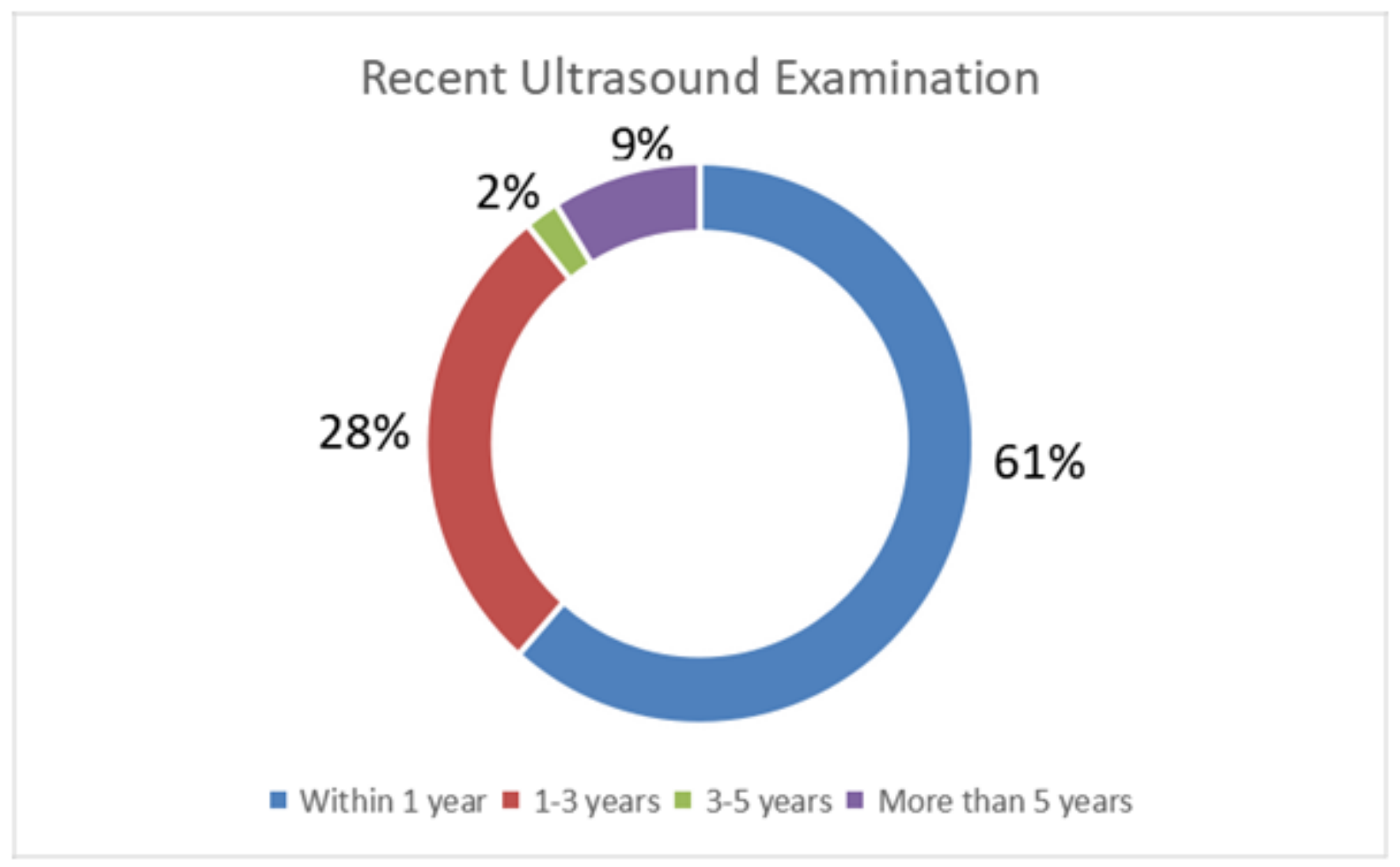

Figure 3

The proportion of the most recent ultrasound review at different time periods.

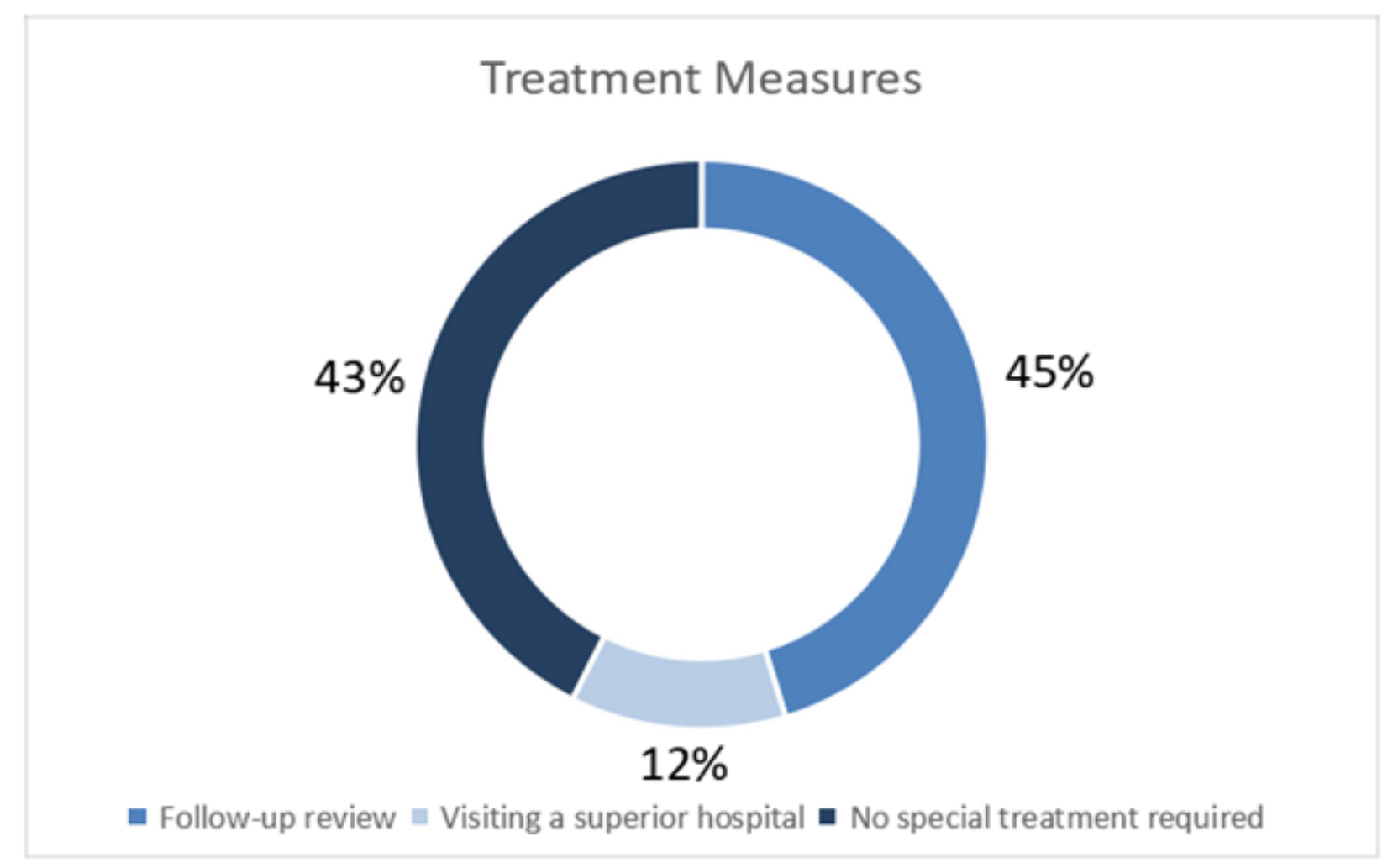


Figure 4

The proportions of different treatment measures 